\title{
Polymorphic tuning of a flexible organic crystal optical waveguide
}

\author{
T. Feiler ${ }^{1,2}$, B. Bhattacharya' ${ }^{1}$ A. Michalchuk ${ }^{1}$, S.-Y. Rhim², V. Schröder²,3, E. List-Kratochvil',3, \\ F. Emmerling ${ }^{1,2}$
}

${ }^{1}$ Federal Institute for Materials Research and Testing (BAM), Richard-Willstätter-Str. 11, 12489 Berlin, Germany, ${ }^{2}$ HumboldtUniversität zu Berlin, Brook-Taylor-Str. 2, 12489 Berlin, Germany, ${ }^{3}$ Helmholtz-Zentrum Berlin für Materialien und Energie GmbH, Hahn-Meitner-Platz 1, 14109 Berlin, Germany

Torvid.Feiler@bam.de

Crystalline molecular materials are usually brittle and are prone to break upon external mechanical force. This fragility poses challenges for their application in next-generation technologies, including sensors, synthetic tissues, and advanced opto-electronics. The recent discovery of mechanical flexibility in single crystals of molecular materials has solved this problem and enable the design of smart flexible device technologies.[1] Mechanical flexibility of organic crystals can be tuned by altering the weak interactions in the crystal structure, for example through polymorphism. Here we report 4-bromo-6-[(6-chloropyridin-2-ylimino)methyl]phenol (BCMPMP) as a promising candidate for future waveguide technologies. It turns out that BCMPMP has two different polymorphs with distinct optical and mechanical properties. Form I shows brittle behavior under mechanical stress and exhibits very weak emission at $605 \mathrm{~nm}\left(\lambda_{\mathrm{ex}}=425 \mathrm{~nm}\right)$ together with a low photoluminescence quantum yield $(\Phi=0.4 \%)$. In contrast, Form II has a large plastic (irreversible bending) regime and a bright emission at $585 \mathrm{~nm}\left(\lambda_{\mathrm{ex}}=425 \mathrm{~nm} ; \Phi=8.7 \%\right)$. Making use of favorable mechanical flexibility and optical properties, form II was explored as a bendable optical waveguide. Light was successfully propagated through a straight-shaped and mechanically deformed BCMPMP crystal. Depending on the light source, active or passive waveguiding could be achieved. So BCMPMP can also be used as a flexible wavelength filter.

[1] Annadhasan, M., Agrawal, A. R., Bhunia, S., Pradeep, V. V., Zade, S. S., Reddy, C. M. \& R. Chandrasekar (2020), Angew Chem Int Ed, 59, 13852-13858.

Keywords: Flexible Crystals; Polymorphs; Waveguides 